\title{
Analysis of two ALMA front end receiver channels using physical optics - art. no. 68930D
}

Conference Paper · February 2008

DOI: $10.1117 / 12.762749$

CITATIONS

0

4 authors:

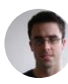

Mark Whale

Universität Bern

8 PUBLICATIONS 8 CITATIONS

SEE PROFILE

Neil Trappe

National University of Ireland, Maynooth

105 PUBLICATIONS 1,651 CITATIONS

SEE PROFILE

Some of the authors of this publication are also working on these related projects:

Project ALMA band 10 View project

Project Herschel-HIFI View project
READS

56

Massimo Candotti

42 PUBLICATIONS 95 CITATIONS

SEE PROFILE

John Anthony Murphy

National University of Ireland, Maynooth

379 PUBLICATIONS 18,894 CITATIONS

SEE PROFILE 


\title{
Analysis of two ALMA Front End Receiver Channels using Physical Optics
}

\author{
Mark Whale ${ }^{(1)}$,Massimo Candotti ${ }^{(1)}$,Neil Trappe ${ }^{(1)}$, Anthony Murphy ${ }^{(1)}$, \\ Andrey Baryshev ${ }^{(2)}$, Victor Belitsky ${ }^{(3)}$ \\ (1) - National University of Ireland, Maynooth, Co. Kildare, Ireland. \\ (2) Space Research Organisation of Netherlands, Landleven 12, Groningen, Netherlands. \\ (3) Chalmers University of Technology, S-412 96 Gothenburg, Sweden.
}

\begin{abstract}
The Atacama Large Millimetre Array will be a single research instrument composed of up to 50 high precision antennas, located at the Chajnantor plain in the district of San Pedro de Atacama, 5000m above sea level. Each ALMA telescope will contain 10 frequency channels/bands, ranging from 30 to $950 \mathrm{GHz}$. Radiation from the secondary reflector is collected to the receivers of each wavelength channel through their accompanying front end optics. We present a full electromagnetic treatment of the front end optics for band $5(163-211 \mathrm{GHz})$ and band $9(602-720 \mathrm{GHz})$. A full quasi optical and physical optics analysis of the band 5 front end optics, using the antenna analysis tool, GRASP9 [1] is presented. Potential optimisation for the system is presented, namely a reflector edge taper and a comparison of two surface geometries. A similar analysis of the band 9 system is presented. Full electromagnetic simulations are compared with cold beam pattern measurements made at the Space Research Organisation of the Netherlands [2, 3]. Analysis of the effect of the polarizing grid is presented, with suggested modifications to improve cross polar levels.
\end{abstract}

Keywords: ALMA, Band 5, Band 9, Quasioptical Analysis \& Verification, GRASP9.

\section{INTRODUCTION}

\subsection{The ALMA Instrument}

The Atacama Large Millimetre Array is an international project to construct a ground based interferometer array to work in the submillimetre/far-infrared region and is considered to be the successor to the present generation of millimetre and sub-millimetre wave interferometers. The ALMA instrument will utilise high resolution radio astronomy techniques applied to the $\mathrm{mm}$ and Submm region, allowing astronomers to observe the cool Universe, determine the chemical composition the molecular gas and dust in star forming regions, observe the redshifted dust continuum emission from galaxies at various epochs of evolution, reveal the kinematics of previously obscured Galactic Nuclei and Quasi-Stellar Objects and obtain high resolution images of cometary nuclei, asteroids and Kuiper Belt Objects along with the planets of the Solar System and their satellites [4].This research is carried out with our collaborators at the Space Research Organisation of the Netherlands, Groningen and the Chalmers Technical University, Gothenburg, Sweden.

The instrument will be comprised of $5012 \mathrm{~m}$ antennas with $25 \mathrm{um}$ surface accuracy and $0.6^{\prime \prime}$ pointing precision. This array of antennas can be arranged into various configurations with maximum and minimum widths of $14 \mathrm{~km}$ and $150 \mathrm{~m}$ respectively. The array will be located $5000 \mathrm{~m}$ above sea level on the Chajnantor plane in the Atacama region of Chile, which will provide excellent atmospheric transparency for the observable millimetre and sub-millimetre wavebands.

\subsection{The ALMA Front End Receivers}

The ALMA instrument will have a frequency coverage of $30 \mathrm{GHz}$ to $950 \mathrm{GHz}$ in 10 dual polarisation bands. Each of these bands has a modular 'plug in' design, and all 10 bands are housed in a single dewar flask, located at the Cassegrain focal plane, meaning that all frequency bands share the same focal plane, removing any need for a selection mirror arrangement. The bands are divided into 3 separate categories; indicative of the requirements of the particular receiver frequency. Category A receivers (bands 1 \& 2) contain 'warm' optics; Category B receivers contain a mixture of 'warm' and 'cold' optics (bands $3 \& 4$ ) and Category C receivers contain entirely 'cold' optics (bands 5 - 10). The work presented in this paper is devoted to the study of two of these front end optics receivers - bands 5 and 9 , which are both Category $\mathrm{C}$ receivers. [3]

Terahertz Technology and Applications, edited by Kurt J. Linden, Laurence P. Sadwick Proc. of SPIE Vol. 6893, 68930D, (2008) · 0277-786X/08/\$18 - doi: 10.1117/12.762749 


\section{APPLICATION}

\subsection{ALMA Band 5 - Introduction}

The design and assembly of this band is the responsibility of the Radio and Space Science Department at the Chalmers University of Technology in Gothenburg, Sweden. Quasioptical and physical optical analysis of this receiver has been conducted at NUI Maynooth. The ALMA Band 5 receiver has a frequency range of $163 \mathrm{GHz}$ to $211 \mathrm{GHz}$ with a central operating frequency of $187 \mathrm{GHz}$. It is comprised of two off-axis ellipsoidal reflectors, which couple the Cassegrain focal plane to the circular corrugated receiver horn. Dual polarisation is achieved in the band with an orthomode transducer at the back of the waveguide. Analysis presented in this paper includes a full quasioptical treatment of the system and physical optics software simulations, which include predictions of aperture efficiency, subreflector edge taper, beam Gaussicity and cross polar levels. Physical optics predictions are also used for a comparison of system efficiency against varying reflector rim diameters and to verify an improvement to system efficiency with a redesign of the ellipsoidal reflector geometries.

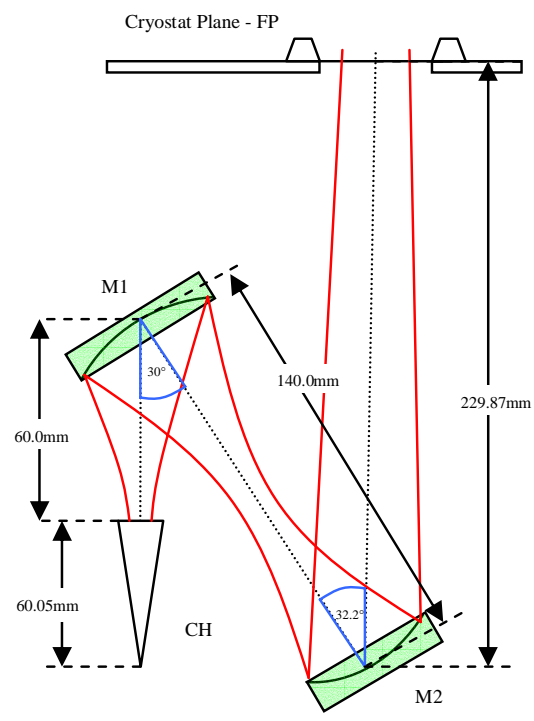

Figure 1 - Band 5 Optical Layout

\subsubsection{Band 5 Parameters}

The parameters listed below in Table 1 were obtained by propagating a fundamental Gaussian beam through an ABCD matrix representation of the system. Using ABCD matrices, the beam parameters can be calculated at any position throughout the system. Using these parameters and the diagram of the system layout, a model of the system was generated in the Physical Optics software GRASP9. The feed for the system is a circular corrugated horn, designed by the research group at Chalmers. Rather than using a fundamental Gaussian field or a truncated Bessel field as an approximation to the source field, a description of the aperture field generated by the corrugated horn was used. This aperture field was generated using a mode-matching scattering matrix software package that was developed at Maynooth called SCATTER. The near and far field descriptions calculated from SCATTER [5] have been verified against various experimental data. 
Table. 1. Band 5 optics parameters for the lower, central and upper frequencies.

\begin{tabular}{|c|c|c|c|c|c|c|c|c|c|}
\hline Frequency (GHz) & & 163 & 187 & 211 & Frequency $(\mathbf{G H z})$ & & 163 & 187 & 211 \\
\hline Horn Diameter (mm) & 9.0 & & & & $\mathrm{Z1}-\mathrm{M} 1$ to $w_{1}(\mathrm{~mm})$ & & 62.119 & 60.338 & 58.716 \\
\hline Horn Axial Length (mm) & 60.0 & & & & $w_{1}$ & & 2.761 & 2.615 & 2.473 \\
\hline Horn Slant Length (mm) & 60.169 & & & & D2 - M1 to M2 (mm) & 140.0 & & & \\
\hline Horn Waist, $w_{0}(\mathbf{m m})$ & & 2.817 & 2.793 & 2.763 & F2 (mm) & 67.192 & & & \\
\hline Horn Waist Offset, $z_{\theta}(\mathrm{mm})$ & & -3.227 & -4.176 & -5.218 & Waist M2, $w_{M 2}(\mathrm{~mm})$ & & 16.731 & 15.763 & 15.07 \\
\hline Waist at Horn Aperture, $w_{H A}$ & & 2.896 & 2.896 & 2.896 & $\theta 2\left(^{\circ}\right)$ & 32.2 & & & \\
\hline D1 - CH to M1 (mm) & 60.05 & & & & $\mathrm{Z2}-\mathrm{M2}$ to $w_{\text {CASS }}(\mathrm{mm})$ & & 236.924 & 235.187 & 234.007 \\
\hline F1 (mm) & 32.756 & & & & $w_{\text {CASS }}(\mathbf{m m})$ & & 11.01 & 9.599 & 8.508 \\
\hline Waist M1, $w_{M I}(\mathrm{~mm})$ & & 13.449 & 12.061 & 11.02 & $w_{S U B}(\mathbf{m m})$ & & 318.832 & 318.832 & 318.832 \\
\hline$\theta 1\left(^{\circ}\right)$ & 30.0 & & & & Edge Taper (dB) & & 12 & 12 & 12 \\
\hline
\end{tabular}

\subsubsection{Geometrical and Quasioptical Reflector Designs}

Physical optics predictions of the Band 5 system were calculated using the GRASP9 optics package, which has long been considered the benchmark software for antenna beam pattern predictions in this wavelength region [1,5]. Two 'versions' of the system were designed; a 'geometrical' and a 'quasioptical' version. These versions refer to the ellipsoidal reflector shapes, where the geometrical design uses geometrical optics to define the mirror surface, and in the quasioptical design the reflector shapes best match the input phase radius of curvature of the incoming beam at the central frequency. The purpose of such a test is valid since each ALMA front end optics cartridge will be or has been designed using geometrical optics, the purpose of which is to allow easier verification across the bands. This comparison with a quasioptical design will reveal any losses in performance neglecting long wavelength effects.
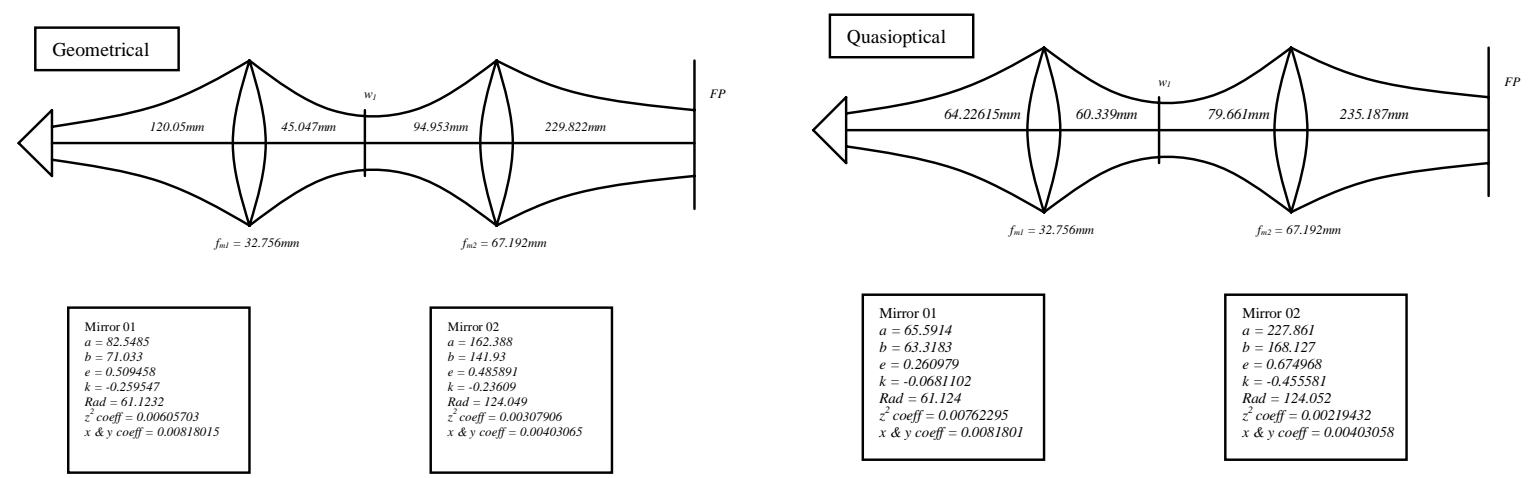

Figure 2 - geometrical and quasioptical beam path calculations - ellipsoidal mirror parameters included were generated and ellipsoidal mirror were generated using $2^{\text {nd }}$ order polynomials.

The ellipsoidal mirrors are described in GRASP9 by the $2^{\text {nd }}$ order polynomial equation,

$$
\frac{x^{2}}{2 \operatorname{Rad}}+\frac{y^{2}}{2 \operatorname{Rad}}+\frac{1+k}{2 \operatorname{Rad}} z^{2}=0
$$

where $k$ is the conic constant and Rad is the radius of curvature. For the geometrical limit, the input and output radii, $R_{i n}$ $R_{\text {out }}$ of the ellipses are determined using the usual geometrical focus equation. For the quasioptical version, they are determined as follows:

$$
R_{\text {in }, \text { out }}=d_{i n, \text { out }}\left(1+\left(\frac{\pi \cdot w_{\text {in,out }}^{2}}{\lambda \cdot d_{i n, \text { out }}}\right)^{2}\right)
$$




\subsubsection{Reflector Rim Truncation Analysis}

As well as a quasioptical and geometrical design comparison, it was also necessary to perform physical optics analysis of the effect of the off-axis reflector rim truncation of the beam for varying mirror size. In designing any optical reflector system, conservation of power is important. One must strive to confine as much power as possible within the reflector area and reduce spillover. In this compact off-axis reflector system, analysis of this power conservation is more important since there are opto-mechanical limits to be considered. The best overall system is a compromise between the maximum power confined within the reflector area and the maximum allowable reflector size within the band 5 cartridge. No actual mechanical structures are finalized as yet. For this analysis, 3 different rim radii were considered. These reflector rim radii are multiples of the lowest frequency beam waist at each reflector; $2 w_{163}, 2.25 w_{163}$ and $2.5 w_{163}$, each representing varying degrees of truncation of the incident beam. The lowest frequency waist was chosen since this represents the largest beam waist across the bandwidth, and is thus considered the upper limit of truncation. The results presented for both the geometrical and quasioptical versions are given for the upper, middle and lower frequencies and each of the 3 reflector rim radii.

\subsubsection{Results}

From the physical optics simulations of the two versions of the Band 5 system, beam pattern measurements were made at three locations, the focal plane which represents the cryostat plane and the geometrical beam waist position, a potential measurement plane which was described by Chalmers to be $600 \mathrm{~mm}$ from the focal plane where future cold beam pattern measurements will be taken and the subreflector plane. Using these beam pattern measurements, several calculations were made determining the performance of the band 5 system in terms of aperture efficiency (coupling efficiency of the output beam to the sky), edge taper at the subreflector (the amount of power confined to within the subreflector area), Gaussicity (how well the output field couples to a fundamental Gaussian beam) and cross polarisation efficiency (how much power present in the cross polar field relative to the co polar field).

\subsubsection{Aperture Efficiency}

Antenna aperture efficiency is represented as the coupling efficiency between the beam at the subreflector vertex plane and a truncated plane wave.

$$
\eta_{a}=\left|\frac{{ }_{A P} \int E_{a}^{*} E_{t p w} d S}{\sum_{A P} \int\left|E_{a}\right|^{2} d S_{A P} \int\left|E_{t p w}\right|^{2} d S}\right|^{2}
$$

where $A P$ represents an integral over the entire aperture plane, $E_{a}$ is the aperture field and $E_{t p w}$ is the ideal truncated plane wave field. The truncated plane wave used here contains a scaled central blockage, to account for the degradation in the aperture efficiency from the central blockage shadowing of the secondary mirror over the primary reflector. As such, this aperture efficiency calculation takes into account the coupling efficiency, spillover efficiency and the blockage efficiency.

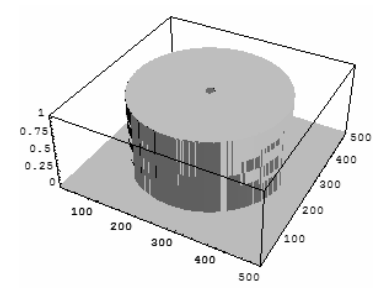

Figure 3 - Ideal truncated plane wave field, 'Top Hat', with scaled central obstruction representing the secondary mirror over the primary mirror. This complex field is used to determine the aperture efficiency - equation 3

Non ideal smoothness of the mirror surfaces from their ideal shape introduces perturbations in the wavefront and thus leads to a decrease in the aperture efficiency. Studies conducted by Ruze [7] show that the actual mirror surface deformations can be statistically modeled assuming that phase errors of a surface point have a mean zero and belong to a Gaussian population of RMS deviation about this mean. Therefore, small-scale surface errors decrease the efficiency and are described by

$$
\eta_{\text {Ruze }}=\operatorname{Exp}\left(-16 \pi^{2} \frac{\left(\sigma_{s}^{2}+\sigma_{p}^{2}\right)}{\lambda^{2}}\right)
$$


Table 2 - Aperture efficiency calculations, determined from subreflector field coupled to obscured truncated plane wave

\begin{tabular}{|c|c|c|c|c|c|c|c|c|c|c|}
\hline Rim & Parameter & $\begin{array}{l}f \\
(\mathrm{GHz})\end{array}$ & GO & QO & $f(\mathrm{GHz})$ & $\mathrm{GO}$ & QO & $f(\mathrm{GHz})$ & $\mathrm{GO}$ & QO \\
\hline \multirow[t]{3}{*}{$2.0 w$} & $\eta_{\mathrm{a}}$ & & 0.822 & 0.838 & & 0.833 & 0.849 & & 0.844 & 0.855 \\
\hline & $\eta_{\text {Ruze }}$ & & 0.934 & 0.934 & & 0.934 & 0.934 & & 0.934 & 0.934 \\
\hline & $\eta_{\text {total }}$ & & 0.768 & 0.783 & & 0.778 & 0.793 & & 0.778 & 0.793 \\
\hline \multirow[t]{3}{*}{$2.25 w$} & $\eta_{\mathrm{a}}$ & & 0.829 & 0.853 & & 0.838 & 0.856 & & 0.846 & 0.859 \\
\hline & $\eta_{\text {Ruze }}$ & 163 & 0.924 & 0.924 & 187 & 0.924 & 0.924 & 211 & 0.924 & 0.924 \\
\hline & $\eta_{\text {total }}$ & & 0.765 & 0.796 & & 0.774 & 0.791 & & 0.782 & 0.794 \\
\hline \multirow[t]{3}{*}{$2.5 w$} & $\eta_{\mathrm{a}}$ & & 0.829 & 0.859 & & 0.838 & 0.859 & & 0.845 & 0.862 \\
\hline & $\eta_{\text {Ruze }}$ & & 0.915 & 0.915 & & 0.915 & 0.915 & & 0.915 & 0.915 \\
\hline & $\eta_{\text {total }}$ & & 0.758 & 0.785 & & 0.767 & 0.786 & & 0.773 & 0.789 \\
\hline
\end{tabular}

\subsubsection{Subreflector Edge Taper}

The edge taper at the subreflector is the relative power density within a specified radius $r$. In this case, the edge taper represents the amount of power confined within the subreflector, which has a radius of $375 \mathrm{~mm}$. For a fundamental Gaussian beam, the edge taper $T_{e}$ is given as:

$$
T_{e}=\operatorname{Exp}\left(-2\left(\frac{r}{w}\right)^{2}\right)
$$

The truncation of the equivalent fundamental Gaussian beam for the subreflector gives an edge taper of $-12 \mathrm{~dB}$ which optimizes the equivalent Truncated Bessel field representing a scalar horn input. However, the maximum aperture efficiency for an unblocked Gaussian illumination is $-10.9 \mathrm{~dB}$ [8]. To determine edge taper for the band 5 system, the field predictions at the vertex of the subreflector were overlaid with a circle with the same radius as the subreflector. The edge taper levels were recorded where the subreflector edge truncated the contour lines of the subreflector field.

Table 3 - Edge taper estimations from truncating circular rim, $r=375 \mathrm{~mm}$, over contour plot of subreflector field.

\begin{tabular}{llll}
\hline Freq $(\mathrm{GHz})$ & Rim & $T_{e}(\mathrm{~dB})-\mathrm{GO}$ & $T_{e}(\mathrm{~dB})-\mathrm{QO}$ \\
\hline \multirow{2}{*}{163} & $2.0 w$ & -11.0 & -10.5 \\
& $2.25 w$ & -10.25 & -9.5 \\
& $2.5 w$ & -10.5 & -9.25 \\
& $2.0 w$ & -11.0 & -10.0 \\
187 & $2.25 w$ & -10.25 & -9.5 \\
& $2.5 w$ & -10.25 & -9.5 \\
& $2.0 w$ & -10.0 & -9.5 \\
& $2.25 w$ & -10.0 & -9.5 \\
& $2.5 w$ & -10.0 & -9.5 \\
\hline
\end{tabular}


Note that some of the edge taper estimates are quoted as averages. This was due to the fact the field from the band 5 front end optics is off-axis, as are all of the ALMA bands, and as such they have varying degrees of angular mismatch to the subreflector, which is perpendicular to the cryostat plane rather than the incident beam of any one band. This is most evident in the geometrical version, owing to poorer focusing ability against the quasioptical version.
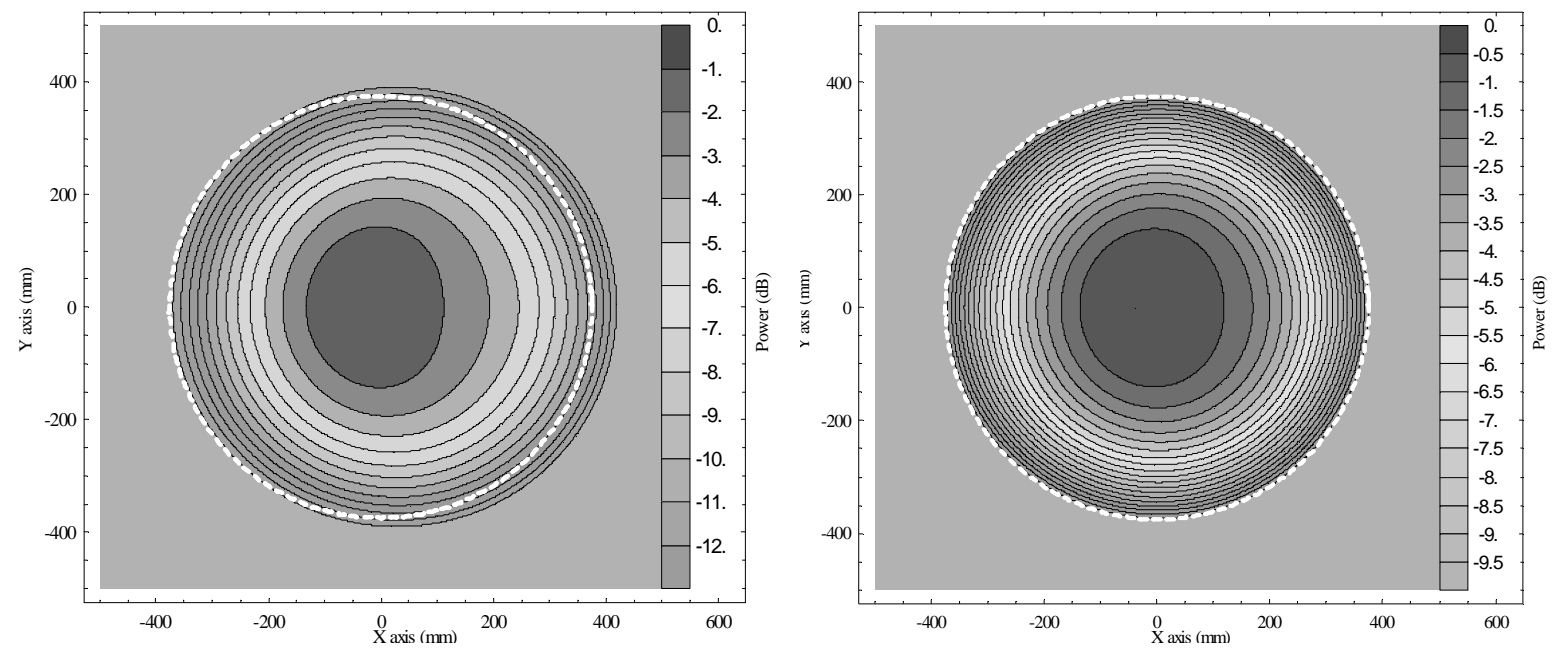

Figure 4 - Sample contour plots of the field at the subreflector (geometrical on left and quasioptical on right), with subreflector rim included (broken white line).

\subsubsection{Cross Polar Efficiency}

Cross polar efficiency is an important factor in off axis reflector systems. It has been shown that off axis paraboloids and ellipsoids increase the levels cross polar power, thus decreasing the amount power conserved to the co polar component [8]. However, for systems of compensating off axis reflectors, such as the band 5 system, any cross polar power created by one mirror should be removes, or 'compensate' for by the second. The levels of cross polar power were measured against co polar power at the focal plane, the measurement plane, and the subreflector plane.

Table 4 - Cross polar levels measured at the focal plane, measurement plane and subreflector plane.

\begin{tabular}{llllllll}
\hline Freq $(\mathrm{GHz})$ & Rim & \multicolumn{3}{c}{$X s P(\mathrm{~dB})-\mathrm{GO}$} & \multicolumn{3}{c}{$X s P(\mathrm{~dB})-\mathrm{QO}$} \\
\cline { 3 - 8 } & & FP & MP & SUB & FP & MP & SUB \\
\multirow{2}{*}{163} & $2.0 w$ & -31.13 & -22.7 & -31.46 & -31.05 & -31.21 & -31.31 \\
& $2.25 w$ & -30.96 & -23.98 & -31.19 & -30.97 & -30.99 & -31.05 \\
& $2.5 w$ & -30.83 & -24.13 & -31.04 & -30.85 & -30.85 & -30.89 \\
& $2.0 w$ & -32.28 & -23.16 & -32.54 & -32.17 & -32.38 & -32.47 \\
187 & $2.25 w$ & -32.09 & -23.78 & -32.43 & -32.21 & -32.25 & -32.32 \\
& $2.5 w$ & -32.01 & -24.39 & -32.35 & -32.13 & -32.14 & -32.21 \\
& $2.0 w$ & -29.17 & -27.08 & -29.23 & -29.19 & -29.25 & -29.30 \\
& $2.25 w$ & -29.07 & -27.46 & -29.16 & -29.12 & -29.15 & -29.19 \\
& $2.5 w$ & -29.02 & -27.79 & -29.1 & -29.07 & -29.08 & -29.11 \\
\hline
\end{tabular}

These cross polar levels match well with the levels as measured from the horn aperture, shown in Table 5.

Table 5 - Cross polar levels measured at the horn aperture for the top, middle and bottom operating frequencies.

\begin{tabular}{llll}
\hline Freq $(\mathrm{GHz})$ & 163 & 187 & 211 \\
\hline$X s P(\mathrm{~dB})$ & -37.40 & -36.43 & -29.42 \\
\hline
\end{tabular}




\subsubsection{Conclusions}

Overall the PO predictions of the Band 5 system have performed as expected. Since the Band 5 system is awaiting construction, comparisons of EM predictions against experimental values are planned. However, these predictions will be taken into consideration when deciding on the exact specifications for the construction of the Band 5 system. The effect of varying rim diameters on the beam has been predicted, and the improvement in system performance with a quasioptical design of the reflector surfaces has been shown. With the quasioptical version of the system, we have seen an improvement in the aperture efficiency levels and an improved symmetrical focusing of the beam on the subreflector. With an increase in rim size for both versions of the system we have seen improved aperture efficiency levels, which is to be expected since an increase in collecting area leads to an increase in conserved power between the aperture and the feed. Cross polar efficiency for both versions is excellent, with levels at the subreflector matching the levels at the horn aperture.

The next step in the analysis process at Maynooth is to determine the effect of the ALMA front end optics dewar flask cryostat window on the beam. Analysis of the effect of such dielectrics on electromagnetic fields is not possible in GRASP9. A new software package called MODAL (Maynooth Optical Design and Analysis Laboratory) which has been developed in Maynooth will be used for these calculations [9].

\subsection{ALMA Band 9 - Introduction}

This high priority band is already well developed, with 9 modules already constructed and tested at the Space Research Organisation of Netherlands (SRON), Groningen. This band has a central frequency of $661 \mathrm{GHz}$, with upper and lower frequencies of $720 \mathrm{GHz}$ and $602 \mathrm{GHz}$ respectively. At this high frequency, orthomode transducers are no longer viable for polarisation separation, and a polarising grid is utilised between after the first reflector, with equivalent optics for both polarisations after the grid. This is the method employed for Bands 7 - 10 in ALMA. The mirrors M41P and M40P are equal reflectors which image each horn, $\mathrm{CH} 1 \mathrm{P}$ and $\mathrm{CHOP}$ to the subreflector focal plane. The blue arrow indicates the direction of polarisation of the $1 \mathrm{P}$ path, and the green arrow indicated the direction of polarisation for the $0 \mathrm{P}$ path.

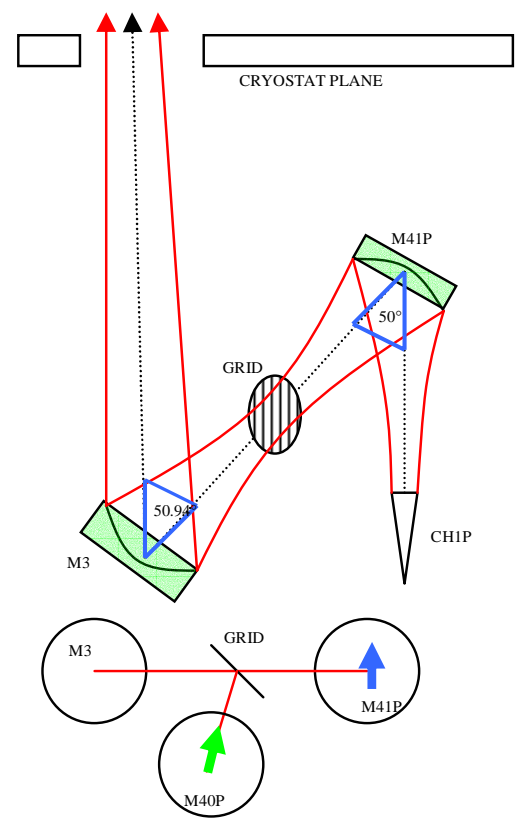

Figure 5 - Band 9 Optical Layout

The LO injection is achieved through dielectric Mylar beam splitters at $45^{\circ}$ in front of the mixer horns. The LO feeds, which are diagonal horns, are coupled to the mixers through pairs of ellipsoidal mirrors. This kind of feed gives an optimum Gaussian coupling of $84 \%$, which is sufficient power coupling for the mixer horn. A full quasi optics and physical optics analysis of the band is presented. In addition to this characterisation of the system, near field beam pattern measurements were performed both at room temperature and with the system placed within the cryogenic cartridge. The experimental data was compared with the physical optics results from GRASP9 and the antenna efficiency and main beam features were retrieved for both experiment and software models. Other work includes a study of the effects of the polarising grid on the output beam, namely the cross polar levels, and a redesign of the ellipsoidal reflector geometries, similar to that performed for band 5 . 


\subsubsection{Band 9 Parameters}

The table below lists the quasioptical parameters of the Band 9 system, determined through the ABCD matrix method, similar to table of parameters for Band 5 (c.f. Table 1). The physical optics model of the system was developed in GRASP9 using these parameters combined with the optical layout shown in Figure 5.

Table. 6. Band 9 optics parameters for the lower, central and upper frequencies.

\begin{tabular}{|c|c|c|c|c|c|c|c|c|c|}
\hline Frequency $(\mathbf{G H z})$ & & 602 & 661 & 720 & Frequency $(\mathbf{G H z})$ & & 602 & 661 & 720 \\
\hline Horn Diameter (mm) & 5.066 & & & & $\mathrm{Z1}-\mathrm{M} 1$ to $w_{1}(\mathrm{~mm})$ & & 45.340 & 44.924 & 44.584 \\
\hline Horn Axial Length (mm) & 15.516 & & & & $w_{1}$ & & 0.954 & 0.885 & 0.824 \\
\hline Horn Slant Length (mm) & 15.722 & & & & D2 - M1 to M2 (mm) & 95.906 & & & \\
\hline Horn Waist, $w_{0}(\mathrm{~mm})$ & & 1.115 & 1.059 & 1.006 & F2 (mm) & 39.41 & & & \\
\hline Horn Waist Offset, $z_{0}(\mathrm{~mm})$ & & -8.364 & -9.089 & -9.735 & Waist M2, $w_{M 2}(\mathrm{~mm})$ & & 8.457 & 8.363 & 8.291 \\
\hline Waist at Horn Aperture, $w_{H A}$ & & 1.630 & 1.630 & 1.630 & $\theta 2\left({ }^{\circ}\right)$ & $50.94^{\circ}$ & & & \\
\hline D1 - CH to M1 (mm) & 44.484 & & & & $\mathrm{Z2}-\mathrm{M} 2$ to $w_{\text {CASS }}(\mathrm{mm})$ & & 149.482 & 149.435 & 149.399 \\
\hline F1 (mm) & 24.862 & & & & $w_{C A S S}(\mathbf{m m})$ & & 2.996 & 2.729 & 2.505 \\
\hline Waist M1, $w_{M 1}(\mathrm{~mm})$ & & 7.595 & 7.381 & 7.214 & $w_{S U B}(\mathrm{~mm})$ & & 317.407 & 317.409 & 317.410 \\
\hline$\theta 1\left(^{\circ}\right)$ & $50^{\circ}$ & & & & Edge Taper (dB) & 12.0 & 12.12 & 12.12 & 12.12 \\
\hline
\end{tabular}

\subsubsection{Band 9 - Quasioptical Analysis and Results}

Electrical field distributions of the two orthogonally polarised beams have been both measured and predicted at the focal plane. Therefore, detailed comparisons of the beam features at precise locations are possible. Room temperature and cooled near field measurements have been achieved with a dedicated amplitude and phase measurement system capable of frequency tuning spanning the entire frequency range of Band 9. Measurements have been taken for both polarisations on a common plane [2]

\subsubsection{Aperture Efficiency}

Aperture efficiency is calculated suing the same method described in Section 3.1.4.1.

Table 8 - Software and experiment aperture efficiency calculations for the $0 \mathrm{P}$ and $1 \mathrm{P}$ polarisations of the band 9 system

\begin{tabular}{llcccc}
\hline Freq $(\mathrm{GHz})$ & Parameter & \multicolumn{2}{c}{ Software } & \multicolumn{2}{c}{ Experimental } \\
\cline { 3 - 6 } & & $0 \mathrm{P}$ & $1 \mathrm{P}$ & $0 \mathrm{P}$ & $1 \mathrm{P}$ \\
\multirow{2}{*}{602} & $\eta_{a}$ & 0.821 & 0.815 & 0.789 & 0.780 \\
& $\eta_{\text {Ruze }}$ & 0.773 & 0.773 & 0.773 & 0.773 \\
& $\eta_{\text {tot }}$ & $\mathbf{0 . 6 3 4}$ & $\mathbf{0 . 6 3 0}$ & $\mathbf{0 . 6 0 9}$ & $\mathbf{0 . 6 0 3}$ \\
& $\eta_{a}$ & 0.786 & 0.783 & 0.776 & 0.798 \\
& $\eta_{\text {Ruze }}$ & 0.733 & 0.733 & 0.733 & 0.733 \\
& $\eta_{\text {tot }}$ & $\mathbf{0 . 5 7 6}$ & $\mathbf{0 . 5 7 4}$ & $\mathbf{0 . 5 6 9}$ & $\mathbf{0 . 5 8 5}$ \\
& $\eta_{a}$ & 0.719 & 0.721 & 0.762 & 0.756 \\
& $\eta_{\text {Ruze }}$ & 0.692 & 0.692 & 0.692 & 0.692 \\
& $\eta_{\text {tot }}$ & $\mathbf{0 . 4 9 7}$ & $\mathbf{0 . 4 9 9}$ & $\mathbf{0 . 5 2 7}$ & $\mathbf{0 . 5 3 0}$ \\
& & & & & \\
\hline
\end{tabular}

\subsubsection{Gaussicity}

From beam pattern measurements and predictions it was possible to compute the fundamental Gaussian beam mode fitting analysis. This analysis is an indication of the beam quality, showing how well the beam couples with a fundamental Gaussian and its dislocation from the nominal position. 
Table 7 - Fundamental Gaussian Beam Mode fitting analysis for two frequencies for both software predictions and experimental measurements.

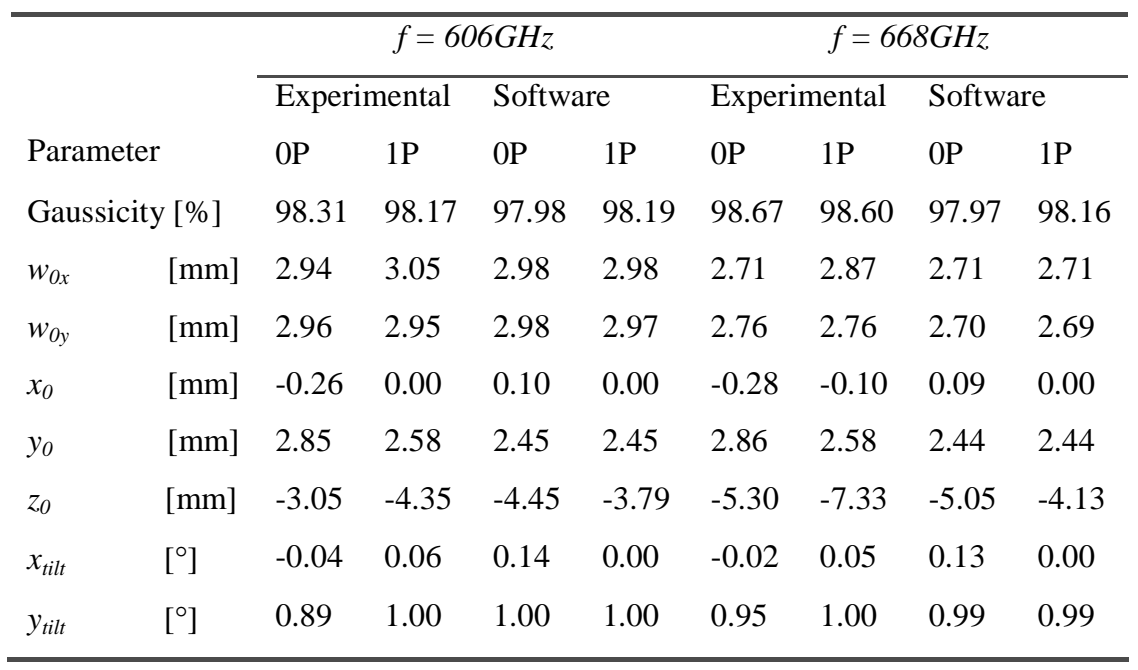

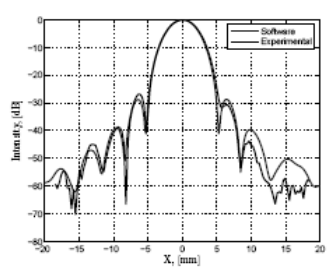

a)

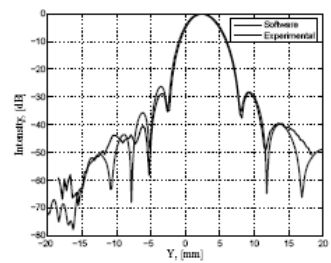

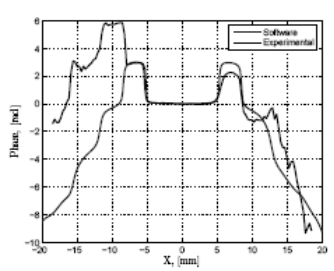

b)

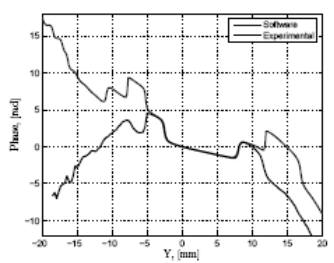

Figure 6 - 1P signal polarisation comparison of software prediction against experiment at the M3 focus - E-Plane cut a) and b) and H-Plane cut c) and d)

\subsubsection{Cross Polar Efficiency}

Cross Polar levels were calculated from EM predictions in GRASP9 and compared against experiment for both the 0P and 1P polarisations. The results are given in Table 9. The levels are given for the central frequency of $661 \mathrm{GHz}$.

Table 9 - Cross polar levels for $\mathrm{t}$ he band 9 system for the $\mathrm{OP}$ and $1 \mathrm{P}$ polarisations

\begin{tabular}{lllll}
\hline Freq $(\mathrm{GHz})$ & \multicolumn{2}{c}{ Software } & \multicolumn{2}{c}{ Experimental } \\
\cline { 2 - 5 } & $0 \mathrm{P}(d B)$ & $1 \mathrm{P}(d B)$ & $0 \mathrm{P}(d B)$ & $1 \mathrm{P}(d B)$ \\
661 & -19.64 & -19.93 & -18.5 & -16.5 \\
\hline
\end{tabular}




\subsubsection{Subreflector Edge Taper}

The predictions of the beam edge taper at the subreflector were calculated in the same way as those presented in Section 3.1.4.2. To measure the edge taper levels using experimental data, the beam pattern measurements at the measurement plane were Fourier transformed to the far-field and scaled to match the size of the subreflector. As with the cross polar levels, results are presented for the central frequency.

Table 10 - Experimental and software comparisons of the beam edge taper at the subreflector for Band 9

\begin{tabular}{lllll}
\hline Freq $(\mathrm{GHz})$ & \multicolumn{2}{c}{ Software } & \multicolumn{2}{c}{ Experimental } \\
\cline { 2 - 5 } & $0 \mathrm{P}(d B)$ & $1 \mathrm{P}(d B)$ & $0 \mathrm{P}(d B)$ & $1 \mathrm{P}(d B)$ \\
661 & -10.0 & -9.5 & -11.5 & -12.0 \\
\hline
\end{tabular}

\subsubsection{Geometrical and Quasioptical Reflector Designs}

A similar analysis to that presented in Section 3.13, where the radii of curvature of the ellipsoidal mirrors were redesigned to exactly match the input phase radius of the incident and reflected central frequency beam; this is different from the current system, where the optics were designed geometrically. Analysis of this new version of the system reveals little or no improvement in the aperture efficiency of the beam to the subreflector. This result is expected since the operating frequency of the band is so high as to be practically in the geometrical limit.

\subsubsection{The Band 9 Polarising Grid - Analysis of Effects on Cross Polarisation}

It has been shown that off axis ellipsoidal or parabolic reflectors will introduce projection effects into the polarisation angle of the incident radiation [10]. (This reflector also radiates a cross-polar component, due to its asymmetric structure). For systems of paired offset reflectors, this projection effect is removed from the output beam, since the cross polarisation rotation introduced by the first reflector is compensated for by the second reflector. However, the band 9 system contains a selective polarising grid located between M41P, M40P and M3. This grid is a standard parallel strip grid and works in reflection for the $0 \mathrm{P}$ polarisation and in transmission for the $1 \mathrm{P}$ polarisation. The projection effects introduced by M41P and M40P means that the polarisation of the input beams are no longer at the correct orientation relative to the grid lines, resulting in an increase in cross polarisation levels after M3.

BAND 9 NOMINAL - GRID ROTATION CROSS POLAR LEVELS

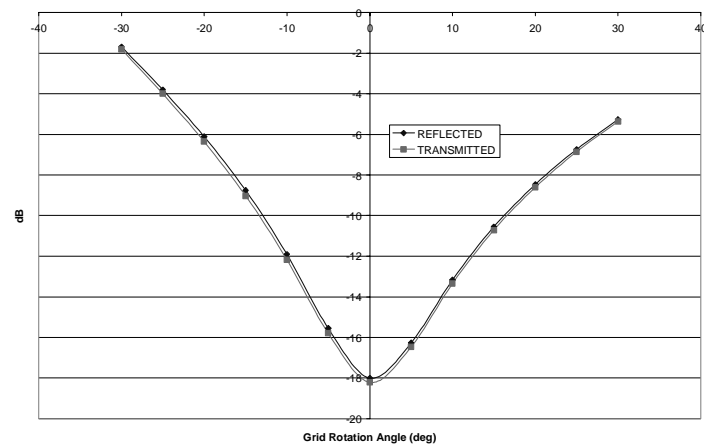

BAND 9 ROTATED - GRID ROTATION CROSS POLAR LEVELS

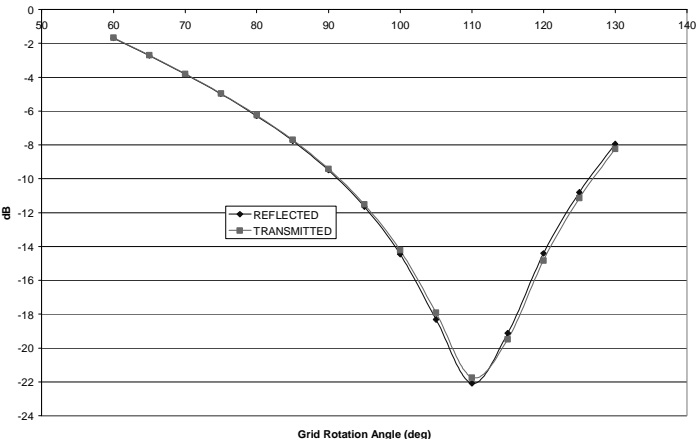

Figure 7 - Cross polar levels as measured at the MP of the band 9 system. The rotated version of the system (right) predicts a reduction in the cross polar levels for a grid rotation of $110^{\circ}$.

Tests were generated in the PO model to determine whether there was an optimal configuration of this system away from the current setup in terms of the cross polar levels. Two versions of the system were tested - the standard set up as described above with the $1 \mathrm{P}$ transmitted polarisation orthogonal to the direction of propagation and the $0 \mathrm{P}$ reflected polarisation parallel to the direction of propagation and the grid lines are vertical; the second setup was where all polarisation directions and grid lines were rotated through $90^{\circ}$. In both setups, the cross polar levels at the output plane were predicted for various rotation angles of the grid to find the optimum angle for transmission and reflection. Predictions reveal an improvement in the cross polar level for the second version of the system, with the grid rotated at 
approximately $110^{\circ}$ relative to its nominal position. Experimental verification of these results at SRON is currently underway.

\subsubsection{Conclusions}

The ALMA band 9 cartridge is the most developed cartridge to date. With nine cartridges currently assembled and rigorously tested, it is performing as expected. The accuracy of the measurement system, together with the electromagnetic model precision led to significant and important descriptions of the beams propagation properties of the beam under study. The combination of software analysis and experimental verification of the optical front end design have proven to be important tools in the assessment of quasioptical system parameters. With the further analysis of the effect of the grid on the output beam cross polar levels presented here and the upcoming experimental verification of the results, it is hoped that the system efficiency will be improved.

Further analysis of the system will include a resolution of the pointing accuracies of the $0 \mathrm{P}$ and $1 \mathrm{P}$ polarisation beams on the subreflector. This analysis will include a full electromagnetic simulation analysis with experimental verification from SRON.

\section{REFERENCES}

1. GRASP9 - General Reflector and Antenna Farm Analysis Software, TICRA, Læderstræde, Copenhagen, Sweden

2. M. Candotti, A. Baryshev, N. Trappe, T. Peacocke, J. Barkhof,, J.A. Murphy, W. Wild, "Verification of the Band 9 ALMA Front End", Proc. of the $7^{\text {th }}$ MINT Millimetre-Wave International Symposium MIS2006 February 15-17 2006, Espoo, Finland

3. H. Rudolf, M. Carter, A. Baryshev, "The ALMA Front End Optics - System Aspects and European Measurement Results", IEEE Trans. on Antennas and Propagation, Vol. 55, No. 11, November 2007

4. "Science with ALMA", ESO website document: http://www.eso.org/projects/alma/science/alma-science.pdf

5. J.A. Murphy, R. Colgan, C. O’Sullivan, B. Maffei, P. Ade, "Radiation Patterns of Multi-Moded Corrugated Horns for Far-Infrared Space Applications" Infrared Physics \& Technology, Vol. 42, pp 515-528, 2001.

6. C. O'Sullivan, E. Atad-Ettedgui, W. Duncan, D. Henry, W. Jellema, J.A. Murphy, N. Trappe, H. van de Stadt, S Withington, G. Yassin, "Far-Infrared Optics Design \& Verification" International Journal of Infrared and Millimetre Waves, Vol. 23, Number 7, 2002.

7. J. Ruze, “Antenna Tolerance Theory - A Review” Proc. IEEE, Vol. 54 p.633-640, April 1966

8. P.F. Goldsmith, "Quasioptical Systems: Gaussian Beam Quasioptical Propagation and Applications”, IEEE Press, Wiley Publishers.

9. J.A. Murphy, S. Withington, "Perturbation Analysis of Gaussian Beam Mode Scattering at Off-Axis Ellipsoidal Mirrors”, Infrared Physics \& Technology, Issue 37, 1996, p 205-209.

10. M.L Gradziel, D. White, S. Withington, J.A. Murphy, "Fast CAD Software for the Optical Design of Long Wavelength Systems" 2005 Joint $30^{\text {th }}$ Intl. Conf. On Infrared and Millimetre Waves.

11. C. Dragone, "New Grids for Improved Polarisation Diplexing of Microwaves in Reflector Antennas", IEEE Trans. on Antennas and Propagation, Vol. 1P-26, No. 3, May 1978. 